\title{
Abscopal Effect After SIRT: It Exists, but How Could We Use It?
}

\author{
Julien Edeline ${ }^{1}$ (D) Yan Rolland ${ }^{2} \cdot$ Etienne Garin $^{3}$
}

Received: 26 August 2020/Accepted: 2 September 2020/Published online: 15 September 2020

(C) Springer Science+Business Media, LLC, part of Springer Nature and the Cardiovascular and Interventional Radiological Society of Europe (CIRSE) 2020

In this volume of CVIR, Powerski et al. present an interesting article studying the incidence of the abscopal effect (AE) following radioembolization (also known as Selective Internal Radiation Therapy or SIRT) of liver metastases [1]. The AE is a rare occurrence following external beam radiotherapy (EBRT). It has been described since the 1950s as the shrinkage of tumors outside of the radiation field, frequently occurring a few weeks or months after delivery of EBRT. This rare event has recently gained much interest, with the documentation of an immunological mechanism supporting it, and with the advent of immunooncology. Indeed, the potential for a synergistic effect between EBRT and immunotherapy led to great expectations, and a number of clinical trials were launched... currently, results are still awaited and the promises are not yet met [2].

Mechanisms underlying the AE are now better described [3]. Radiotherapy induces immunogenic cell death, leading to better recognition of the tumor cells by the immune system. Moreover, radiotherapy can stimulate the production of cytokines (like interferon-beta) that could stimulate

Julien Edeline

j.edeline@ rennes.unicancer.fr

Yan Rolland

y.rolland@rennes.unicancer.fr

Etienne Garin

e.garin@ rennes.unicancer.fr

1 Medical Oncology, Centre Eugène Marquis, av bataille Flandres Dunkerque, 35042 Rennes, France

2 Interventional Radiology, Centre Eugène Marquis, Rennes, France

3 Nuclear Medicine, Centre Eugène Marquis, Rennes, France anticancer immune response, as well as the upregulation of MHC (major histocompatibility complex) class I. Another potential interesting interaction between radiotherapy and immunotherapy is the upregulation of PD-L1 (programmed death-ligand 1) following radiotherapy, which could be an escape mechanism but also an opportunity for combination. However, the overall effect of radiotherapy on the immune microenvironment is complex, and clearly depends on the context and on the radiotherapy regimen used. Indeed, the dose and fractionation used was shown to have a major impact for the potential of AE [4]. This was, to our knowledge, only studied in the context of EBRT. How the protracted delivery of SIRT, which is very different from EBRT from a radiobiological perspective, will also have potential for AE was uncertain.

Powerski et al. thus did an important work, trying to elucidate whether AE could arise following SIRT. They applied a stringent methodology to assure that the shrinkage seen was not related to bilateral SIRT or by concomitant systemic treatment, which explain the attrition in the number of studied patients. Their main result confirms what is known after EBRT: AE can appear, but only at a very low frequency: 1 case was certain over 96 patients, and 3 other cases were controversial, leading to a rough estimate of $1-5 \%$ of occurrence of AE following SIRT. This is quite rare, but is in the range of what is expected after EBRT, thus confirming that SIRT is a modality that can elicit AE.

Will this AE be of any clinical use? Clearly, this would be the case only by using SIRT in combination with immunotherapy. This is already the case in ongoing trials (such as those reported in clinicaltrials.gov: NCT03099564 or NCT03380130). However, such trials being single arm, it will be difficult to clearly demonstrate that any positive 
effect was related to synergy between treatments. Another potential benefit of the combination will be the different effect of the treatments: while SIRT might help for the local control, immunotherapy might help for the systemic control. In some settings (large tumors with vascular involvement), local control might still be of paramount importance, even if systemic treatment improved their results. Conversely, we raised some concern about potential harms: As a radiation therapy, SIRT also exerts toxicity toward lymphocytes, with frequent and profound decrease in their counts [5]. Whether this will impede any synergy is unknown.

In conclusion, this work reinforces the rationale for the clinical trials of combination of SIRT with immunotherapy. However, only the results of trials demonstrating additive effects will be able to put the AE into clinical use.

Funding No funding was needed for this commentary.

\section{Compliance with Ethical Standards}

Conflict of interest JE, YR and EG acted as consultant for BTG and Boston scientific. JE and EG received research funding from BTG.

\section{References}

1. Powerski M, Drewes R, Omari J, Relja B, Surov A, Pech M. Intrahepatic abscopal effect following radioembolization of hepatic metastases. cardiovascular and interventional radiology. 2020; https://doi.org/10.1007/s00270-020-02612-4.

2. Xing D, Siva S, Hanna GG. The abscopal effect of stereotactic radiotherapy and immunotherapy: fool's gold or El dorado? Clin Oncol (R Coll Radiol). 2019;31:432-43.

3. Rodríguez-Ruiz ME, Vanpouille-Box C, Melero I, Formenti SC, Demaria S. Immunological mechanisms responsible for radiationinduced abscopal effect. Trends Immunol. 2018;39:644-55.

4. Buchwald ZS, Wynne J, Nasti TH, Zhu S, Mourad WF, Yan W, et al. Radiation, immune checkpoint blockade and the abscopal effect: a critical review on timing Dose and Fractionation. Front Oncol. 2018;8:612.

5. Estrade F, Lescure C, Muzellec L, Pedrono M, Palard X, Pracht M, et al. Lymphocytes and neutrophil-to-lymphocyte ratio variations after selective internal radiation treatment for HCC: a retrospective cohort study. Cardiovasc Intervent Radiol. 2020;43:1175-81.

Publisher's Note Springer Nature remains neutral with regard to jurisdictional claims in published maps and institutional affiliations. 